\title{
ZnPP reduces autophagy and induces apoptosis, thus aggravating liver ischemia/reperfusion injury in vitro
}

\author{
YUN WANG $^{1 *}$, XUANXUAN XIONG ${ }^{2 *}$, HAO GUO ${ }^{1}$, MINGBO WU $^{2}$, XIANGCHENG LI ${ }^{3}$, \\ YUANCHAO HU ${ }^{1}$, GUANGWEI XIE ${ }^{1}$, JIAN SHEN ${ }^{4}$ and QINGZHONG TIAN ${ }^{1}$ \\ ${ }^{1}$ Department of Oncological Surgery 2, Xuzhou City Central Hospital, The Affiliated Hospital of Southeast University \\ Medical School (Xuzhou), The Tumor Research Institute of Southeast University (Xuzhou), Xuzhou, Jiangsu 221009; \\ ${ }^{2}$ Department of Gastroenterology 2, Xuzhou City Central Hospital, The Affiliated Hospital of Southeast University \\ Medical School (Xuzhou), Xuzhou, Jiangsu 221009; ${ }^{3}$ Key Laboratory of Living Donor Liver Transplantation, \\ Ministry of Public Health, Liver Transplantation Center, The First Affiliated Hospital of Nanjing Medical University, \\ Nanjing, Jiangsu 210029; ${ }^{4}$ Department of General Surgery, The Second Affiliated Hospital \\ of Nanjing Medical University, Nanjing, Jiangsu 210011, P.R. China
}

Received May 25, 2014; Accepted September 18, 2014

DOI: $10.3892 /$ ijmm.2014.1968

\begin{abstract}
There is growing evidence indicating that autophagy plays a protective role in liver ischemia/reperfusion (IR) injury. Heme oxygenase-1 (HO-1) can also prevent liver IR injury by limiting inflammation and inducing an anti-apoptotic response. Autophagy also plays a crucial role in liver IR injury. The aim of the present study was to investigate the role of HO-1 in liver IR injury and the association between HO-1, autophagy and apoptotic pathways. IR simulation was performed using buffalo rat liver (BRL) cells, and HO-1 activity was either induced by hemin (HIR group) or inhibited by zinc protoporphyrin (ZnPP) (ZIR group). In the HIR and ZIR group, the expression of HO-1 and autophagy-related genes [light chain 3-II (LC3-II)] was assessed by RT-qPCR and the protein expression of caspases, autophagy-related genes and genes associated with apoptotic pathways (Bax) was detected by western blot anlaysis. The results of RT-PCR revealed the genetically decreased expression of HO-1 and autophagy-related genes in the ZIR group. Similar results were obtained by western blot analysis and immunofluorescence. An ultrastructural analysis revealed
\end{abstract}

Correspondence to: Dr Qingzhong Tian, Department of Oncological Surgery 2, Xuzhou City Central Hospital, The Affiliated Hospital of Southeast University Medical School (Xuzhou), The Tumor Research Institute of Southeast University (Xuzhou), 199 Jiefang South Road, Xuzhou, Jiangsu 221009, P.R. China

E-mail: tqz190109@gmail.com

*Contributed equally

Abbreviations: IR, ischemia/reperfusion; HO-1, heme oxygenase-1; ZnPP, zinc protoporphyrin; LC3-II, light chain 3-II; BRL, buffalo rat liver; HBSS, Hank's balanced salt solution

Key words: heme oxygenase-1, autophagy, ischemia/reperfusion injury, mitochondrial apoptotic pathways a lower number of autophagosomes in the ZIR group; in the HIR group, the number of autophagosomes was increased. The expression of Bax and cytosolic cytochrome $c$ was increased, while that of Bcl-2 was decreased following treatment of the cells with ZnPP prior to IR simulation; the oppostie occurred in the HIR group. Cleaved caspase-3, caspase- 9 and poly(ADPribose) polymerase (PARP) protein were activated in the IR and ZIR groups. The disruption of mitochondrial membrane potential was also observed in the ZIR group. In general, the downregulation of HO-1 reduced autophagy and activated the mitochondrial apoptotic pathway.

\section{Introduction}

Autophagy, or cellular self-digestion, is an important pathophysiological process of cell development, differentiation, survival and homeostasis. It involves the degradation of cellular proteins and organelles lying outside the membrane, which originate from the outer membrane of the mitochondria. Lysosomes participate in the formation of the autophagosome, which engulfs small molecules and nutrients and then recycles them for energy production (1-3).

A number of studies have demonstrated that autophagy plays an essential role in a number of hepatic diseases (1-3). It is known that autophagy can play a protective role during nutrient starvation, and it has been shown that autophagy can be induced in liver cells due to lack of nutrition or under hypoxic conditions (1). Hypoxic cell injury is aggravated in the liver that has suffered occlusion of the portal triad and following the return of blood flow delivery $(4,5)$. It has also been found that autophagy can alleviate cell injury by limiting necrosis through cellular self-digestion, reducing oxygen consumption or regulating cell apoptosis (6-8).

Zinc protoporphyrin ( $\mathrm{ZnPP})$ is a compound found in red blood cells when heme production is inhibited by the lack of iron or due to other reasons, and it is an intermediate of glycine and succinyl-CoA that is produced during heme biosynthesis (9). 
$\mathrm{ZnPP}$ inhibits heme oxygenase (HO), the rate-limiting enzyme in the heme degradation pathway. It has been demonstrated that the induction of heme oxygenase-1 (HO-1) attenuates liver cell injury, and that preconditioning with protoporphyrin IX zinc accentuates liver damage (10). A previous study reported that HO-1 attenuates liver injury by inducing autophagy and inhibiting cell apoptosis, and that a decrease in HO-1 activity increases cell apoptosis and thus accentuates liver injury (11). Therefore, it may be of interest to investigate the role of $\mathrm{ZnPP}$ in liver ischemia/reperfusion (IR) injury. ZnPP may play a role in hepatocyte injury by downregulating HO-1 and reducing autophagy, or by inducing apoptosis. However, the mechanisms involved, as well as the association between HO-1 and autophagy and cell apoptotic pathways have not yet been fully elucidated. Increased HO-1 expression may induce autophagy to alleviate liver injury. We thus hypothesized that $\mathrm{ZnPP}$, a hemin inhibitor, may downregulate $\mathrm{HO}-1$ expression, which may subsequently reduce autophagy and induce apoptosis, aggravating hepatocyte injury.

\section{Materials and methods}

Reagents and materials. Dulbecco's modified Eagle's medium (DMEM), penicillin, streptomycin, 10\% heat-inactivated fetal bovine serum (FBS) and pancreatic enzymes were purchased from Gibco (Grand Island, NY, USA). Protoporphyrin IX zinc, mineral oil and Hank's balanced salt solution (HBSS) were from Sigma-Aldrich (St. Louis, MO, USA). Antibodies against $\beta$-actin, HO-1, caspase- 3 , caspase-8, caspase-9, Bax, Bcl-2, poly(ADP-ribose) polymerase (PARP) and cleaved PARP were from Abcam (Cambridge, UK). Cytochrome $c$ and light chain 3-II (LC3-II) antibodies were obtained from Novus Biologicals (Littleton, CO, USA). The following reagents were also used: the JC-1 mitochondrial membrane potential assay kit RIPA lysis buffer (Beyotime, Haimen, China), enhanced chemiluminescence (ECL), protease inhibitor (Pierce Biotechnology, Inc., Rockford, IL, USA), polyvinylidene difluoride membranes (Millipore, Bedford, MA, USA), horseradish peroxidase (HRP)-conjugated secondary antibody (Beijing Zhongshan Golden Bridge Biotechnology Co., Ltd., Beijing, China), TRIzol reagent (Invitrogen Life Technologies, Carlsbad, CA, USA), PrimeScript RT Master Mix and the SYBR Premix Ex Taq ${ }^{\text {TM }}$ real-time PCR kit (Takara Bio, Inc., Shiga, Japan).

Cell culture and treatment. We purchased the buffalo rat liver (BRL) cells from the cell bank of the Chinese Academy of Sciences (Shanghai, China). The cells were cultured in DMEM plus $100 \mathrm{U} / \mathrm{ml}$ penicillin, $100 \mathrm{U} / \mathrm{ml}$ streptomycin and $10 \%$ FBS. The cells were seeded in 6-well plates for treatments, western blot analysis and cell viability analysis.

The cells were divided into 6 groups: i) the control group, in which the cells were cultured in DMEM without any treatment; ii) the IR (IR simulation) group, in which the cells were treated with mineral oil ( $1 \mathrm{ml} /$ well) for $1 \mathrm{~h}$ initially, which simulated ischemia, and then the cells were washed with PBS before the return of nutritional and oxygen supply by the replacement of DMEM for 3, 6 and $12 \mathrm{~h}$, as previously described $(12,13)$; iii) the ZIR (ZnPP treatment prior to IR simulation) group, in which the cells were pre-treated with DMEM without $10 \%$ heat-inac- tivated FBS in the presence of $20 \mu \mathrm{mol} / 1 \mathrm{ZnPP}$ for $12 \mathrm{~h}$, then the cells were washed twice with PBS, immersed in mineral oil, and cultured in DMEM for 3, 6 and $12 \mathrm{~h}$, as previously described (14); iv) the SIR (starvation prior to IR simulation) group, in which the cells were cultured in HBSS medium with $\mathrm{Ca}^{2+}$ and $\mathrm{Mg}^{2+}$ supplemented with $10 \mathrm{mM}$ HEPES $(1 \mathrm{ml} /$ well $)$ for $0.5 \mathrm{~h}$ to induce autophagy, then the cells were washed with PBS before being immersed in mineral oil, and then the cells were finally washed twice with PBS and cultured in DMEM for 3, 6 and $12 \mathrm{~h}$, as previously described (15); v) the HIR (hemin treatment prior to IR simulation) group, in which the cells were pre-treated with DMEM without $10 \%$ heat-inactivated FBS in the presence of $20 \mu \mathrm{mol} / 1$ hemin (HO-1 inducer) for $12 \mathrm{~h}$, then washed twice with PBS, immersed in mineral oil and cultured in DMEM for 3, 6 and $12 \mathrm{~h}$, as previously described (14); and vi) the ZSIR (ZnPP treatment then starvation prior to IR simulation), in which the cells were pre-treated with DMEM without $10 \%$ heat-inactivated FBS in the presence of $20 \mu \mathrm{mol} / 1 \mathrm{ZnPP}$ for $12 \mathrm{~h}$, and washed twice with PBS, then the cells were cultured in HBSS medium with $\mathrm{Ca}^{2+}$ and $\mathrm{Mg}^{2+}$ supplemented with $10 \mathrm{mM}$ HEPES ( $1 \mathrm{ml} /$ well) for $0.5 \mathrm{~h}$ to induce autophagy, and then the cells were washed with PBS before being immersed in mineral oil, and finally washed twice with PBS and cultured in DMEM for 3,6 and $12 \mathrm{~h}$.

Reverse transcription-quantitative polymerase chain reaction $(R T-q P C R)$. Total RNA was isolated from the cells and purified using TRIzol reagent. cDNA was synthesized using the PrimeScript RT Master Mix in a $10-\mu 1$ reaction mixture. According to the SYBR Premix Ex Taq real-time PCR kit, we used cDNA $(2 \mu \mathrm{l})$ as a template in a $20-\mu \mathrm{l}$ reaction. Primers were synthesized by Invitrogen (Shanghai, China). The primers for HO-1 were 5'-GTCAAGCACAGGGTGACAGA-3' (sense) and 5'-CTGCAGCTCCTCAAACAGC-3' (antisense). The following primers were used for the detection of LC3-II expression: 5'-GAGCTTCGAACAAA GAGTGGA-3' (sense) and 5'-CTTCTCACCCTTGTATCG CTCTA-3' (antisense). $\beta$-actin was used as the reference gene in our experiment, and the primers for $\beta$-actin were 5'-TCACCCACACTGTGCCC ATCTACGA-3' (sense) and 5'-CAGCGGAACCGCTCATTG CCAATGG-3' (antisense). For RT-PCR, we used the following cycles: $95^{\circ} \mathrm{C}$ for $30 \mathrm{sec}, 40$ cycles of $95^{\circ} \mathrm{C}$ for $5 \mathrm{sec}, 60^{\circ} \mathrm{C}$ for $31 \mathrm{sec}$ and the dissociation stage: $95^{\circ} \mathrm{C}$ for $15 \mathrm{sec}, 60^{\circ} \mathrm{C}$ for $1 \mathrm{~min}$ and $95^{\circ} \mathrm{C}$ for $15 \mathrm{sec}$.

Western blot analysis. The cells were treated as described above. The cells were then cultured and homogenized in RIPA lysis buffer in the presence of $1 \%(\mathrm{v} / \mathrm{w})$ protease inhibitor. Subsequently, we shook the mixture at $4^{\circ} \mathrm{C}$ for $1 \mathrm{~h}$ and removed the insoluble matter by centrifugation at $40,000 \mathrm{x} g$ at $4^{\circ} \mathrm{C}$ for $1 \mathrm{~h}$. For the analysis of cytochrome $c(16)$, we cultured the cell pellets in the HEPES buffer containing $250 \mathrm{mM}$ sucrose, homogenization by a 22 -gauge needle, and then the homogenate was centrifuged at $800 \mathrm{x}$ g at $4^{\circ} \mathrm{C}$ for $15 \mathrm{~min}$. The supernatants were centrifuged at $10,000 \mathrm{x} \mathrm{g}$ for $15 \mathrm{~min}$ at $4^{\circ} \mathrm{C}$. Finally, we collected the mitochondrial pellets and aliquots of the supernatant (cytosolic fraction). The total protein concentration was determined using the bicinchoninic acid protein assay kit. All proteins were mixed with loading buffer before being resolved on a $12 \%$ sodium dodecyl sulfate polyacrylamide gel electrophoresis 


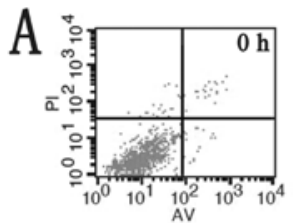

IR Simulation

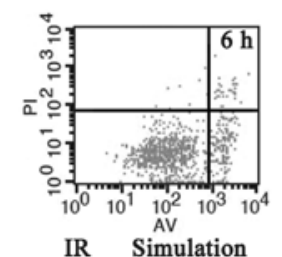

$\mathrm{B}$

IR Simulation

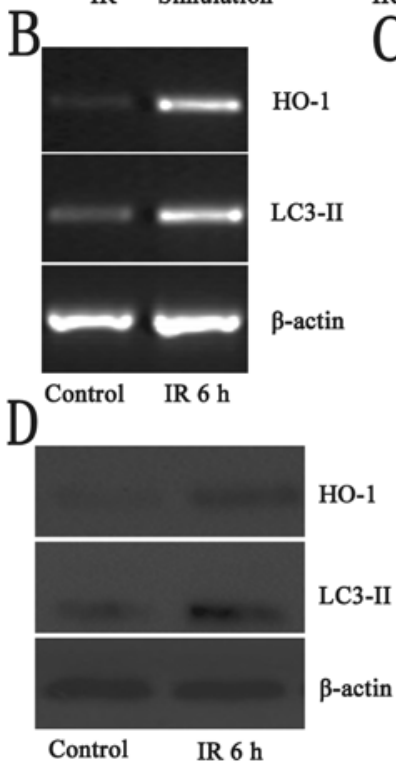

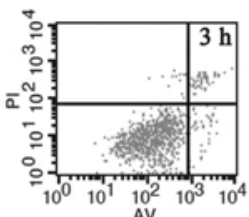

IR Simulation

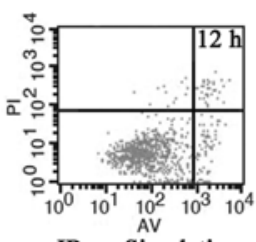

IR Simulation

C
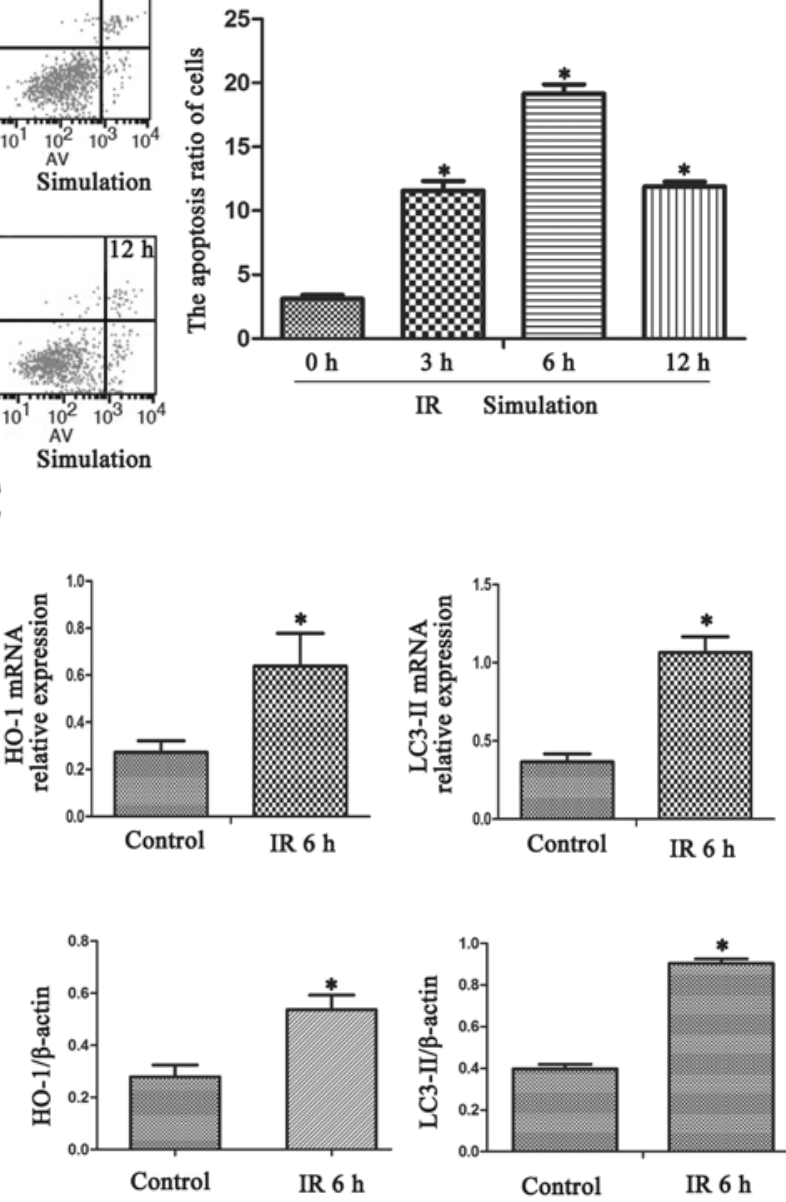

Figure 1. Ischemia/reperfusion (IR) simulation of buffalo rat liver (BRL) cells increased hepatocyte apoptosis, and induced the expression of heme oxygenase-1 (HO-1) and increased autophagy. (A) IR simulation of BRL cells increased hepatocyte apoptosis; the highest apoptotic rate was observed in the 6-h group. (B) IR simulation of BRL cells enhanced the mRNA expression of HO-1 and light chain 3-II (LC3-II) in the 6-h group, as shown by RT-qPCR. (C) IR simulation of BRL cells enhanced the mRNA expression of HO-1 and LC3-II in the 6-h group. (D) IR simulation of BRL cells increased HO-1 and LC3-II protein expression in the 6-h group, as shown by western blot analysis ( $\mathrm{P}<0.05$ compared with the control group).

gel, and this gel was subsequently transferred onto polyvinylidene difluoride membranes at $250 \mathrm{~mA}$ for $2 \mathrm{~h}$. Subsequently, the membranes were blocked in 5\% milk for $1 \mathrm{~h}$ and incubated overnight with primary antibodies (HO-1, LC3-II, $\beta$-actin, caspase-3, caspase-8, caspase-9, cytochrome $c$, Bax, Bcl-2, PARP and cleaved PARP) in $5 \%$ milk. $\beta$-actin protein was used as a control. The membranes were washed the following day in TBS Tween-20 (TBST) for $30 \mathrm{~min}$, and were then incubated with HRP-conjugated secondary antibody $(1: 4,000)$ for $1 \mathrm{~h}$ and washed in TBST again before being visualized using an ECL detection kit. Each experiment was repeated at least 3 times, and the gray value of each protein was measured using Image-Pro Plus 6.0 software for statistical analysis.

Electron microscopic analysis. The cells were plated on 6-well plates, and were harvested with pancreatic enzymes before being fixed in $2 \%$ glutaraldehyde with $0.1 \mathrm{~mol} / 1 \mathrm{PBS}(\mathrm{pH} 7.4$ ). For ultrastructural examination, the cells were post-fixed with $2 \%$ osmium tetroxide $\left(\mathrm{OsO}_{4}\right)$ and were dehydrated through a graded series of ethanol; the cells were then embedded in molds and incubated at $37^{\circ} \mathrm{C}$ overnight. Subsequently, ultrathin sections were made using an ultramicrotome and stained with uranyl acetate and lead citrate. The sections were viewed udner an JEOL JEM 1010 transmission electron microscope (The basic medical laboratory of Nanjing Medical University, Nanjing, China).

Immunofluorescence. The BRL cells were plated on 6-well plates and then following treatment, were fixed on coverslips with $4 \%$ paraformaldehyde for $20 \mathrm{~min}$. After rinsing with PBS 4 times (10 min each time), the cells were blocked in 10\% BSA for $1 \mathrm{~h}$ and incubated with primary antibody (LC3-II in 10\% BSA) overnight. The following day, the cells were rinsed with PBS again before being cultured with secondary antibody $(1: 100)$ for $1 \mathrm{~h}$ at $37^{\circ} \mathrm{C}$. Subsequently, the nuclei were stained with 4',6-diamidino-2-phenylindole (DAPI) and autophagy was monitored. Ten visual fields were randomly selected, and the optical density of each visual field was measured and a semi-quantitative analysis using Image-Pro Plus 6.0 software was performed.

Analysis of apoptosis. The apoptosis of the BRL cells was determined by double staining with Annexin V-fluorescein isothiocyanate (FITC) and propidium iodide (PI). The cells $\left(5 \times 10^{5}\right)$ were washed twice with ice-cold PBS, and were then resuspended in $400 \mu 1$ of binding buffer. Subsequently, 

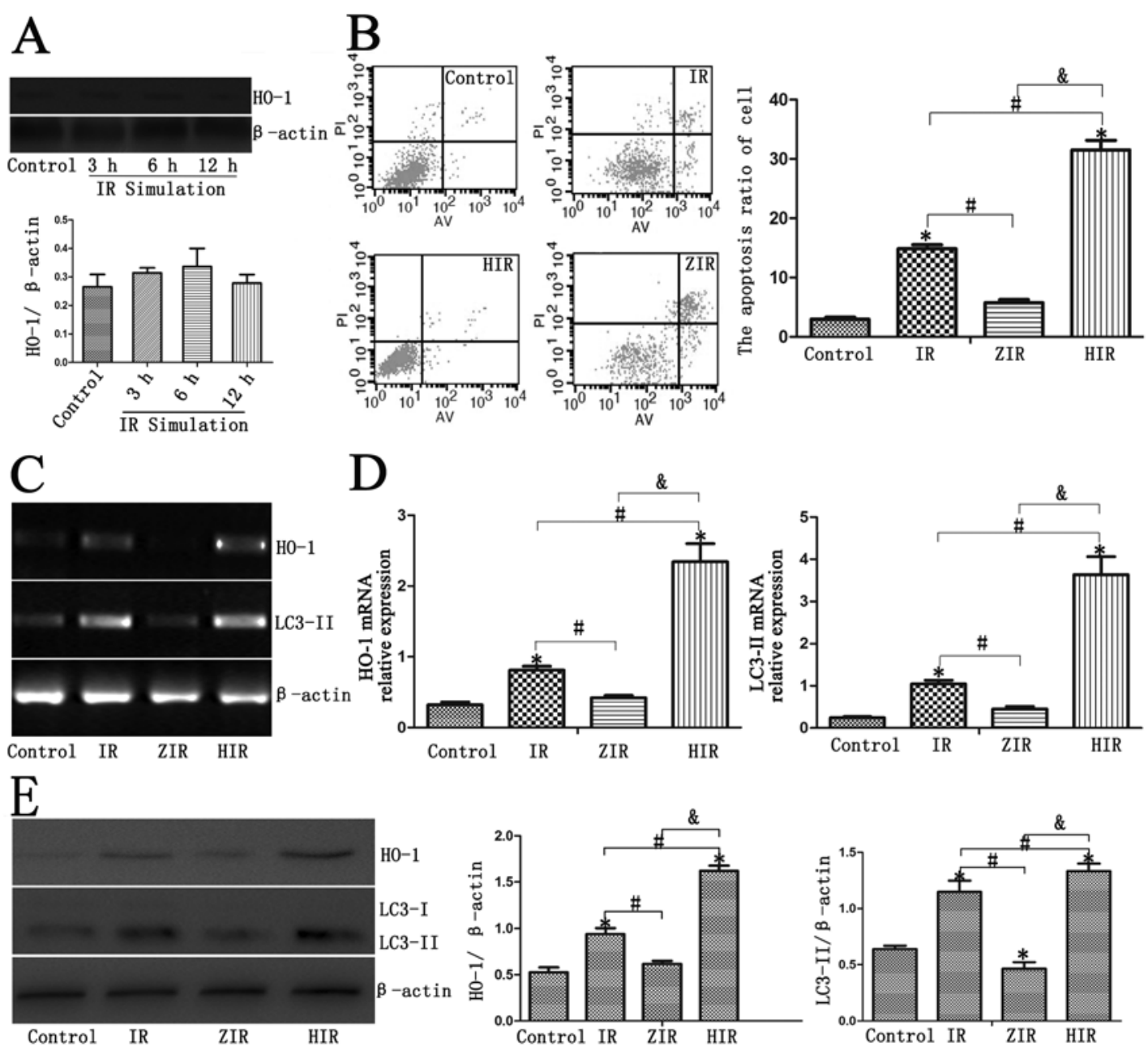

Figure 2. The upregulation of heme oxygenase-1 (HO-1) and autophagy-related genes [light chain 3-II (LC3-II)] reduced hepatocyte apoptosis; however, pretreatment with Zinc protoporphyrin (ZnPP) decreased the protein expression of both HO-1 and LC3-II. (A) Ischemia/reperfusion (IR) simulation induced HO-1 expression. (B) Treatment with ZnPP prior to IR simulation increased the cell apoptotic rates compared with the control and IR groups; however, treatment with hemin prior to IR simulation decreased the cell apoptotic rates compared with the control and IR groups. (C and D) Treatment of buffalo rat liver (BRL) cells with ZnPP prior to IR simulation decreased the mRNA expression of HO-1 and LC3-II; treatment with hemin prior to IR simulation increased the mRNA expression of HO-1 and LC3-II. (E) HO-1 and LC3-II expression was inhibited in the BRL cells by treatment with ZnPP prior to IR simulation; however, their expression was increased by treatment with hemin. These results were verified by statistical analysis of the spatial distribution of gray values (" $\mathrm{P}<0.05$ compared with the control group, ${ }^{,} \mathrm{P}<0.05$ compared with the IR group, ${ }^{\circledR} \mathrm{P}<0.05$ compared with the HIR group).

$5 \mu \mathrm{l}$ of Annexin V-FITC and $5 \mu \mathrm{l}$ of PI were added followed by incubation for 5-15 min in the dark at $4^{\circ} \mathrm{C}$. The samples were analyzed using a FACSCalibur flow cytometer (BD Biosciences, San Jose, CA, USA) using Cell Quest software (BD Biosciences).

Mitochondrial membrane potential assay. A JC-1 mitochondrial membrane potential assay kit was used. Briefly, after the cells were washed with PBS, JC-1 liquid dye was added to each well $\left(1 \mathrm{ml} /\right.$ well), and the cells were incubated at $37^{\circ} \mathrm{C}$ for $20 \mathrm{~min}$. Subsequently, the cells were washed with $1 \mathrm{X}$ dyeing buffer twice, and were cultured in DMEM. Afterwards, the cells were observed under a fluorescence microscope (The basic medical laboratory of Nanjing Medical University).

Statistical analysis. Data are expressed as the means \pm standard deviation. The parameters were analyzed by analysis of variance (ANOVA) and a $\mathrm{Q}$ test and Student's t-test. A value of $\mathrm{P}<0.05$ was considered to indicate a statistically significant difference. SPSS 11.0 software was used in all statistical analyses.

\section{Results}

IR simulation in BRL cells increases hepatocyte apoptosis, and induces HO-1 expression and autophagy. We detected hepatocyte apoptosis in the different IR simulation groups. The highest apoptotic rates were observed in the 6-h group (Fig. 1A; $\mathrm{P}<0.05)$. Thus, the 6-h group was used for our research. RT-qPCR was peformed using cDNA synthesized from different groups of BRL cells as mentioned above. IR simulation enhanced the mRNA expression of HO-1 and LC3-II in the 6-h group (Fig. 1B and C; $\mathrm{P}<0.05$ ). As shown by western blot analysis, the highest protein expression of HO-1 and LC3-II was observed in the 6-h group, (Fig. 1D; P<0.05).

ZnPP treatment prior to IR simulation increases hepatocyte apoptosis, inhibits HO-1 expression and reduces autophagy. Treatment with ZnPP prior to IR simulation (ZIR group) increased the cell apoptotic rates compared with the control and IR groups (Fig. 2B; P $<0.05$ ). We found that $\mathrm{ZnPP}$ 

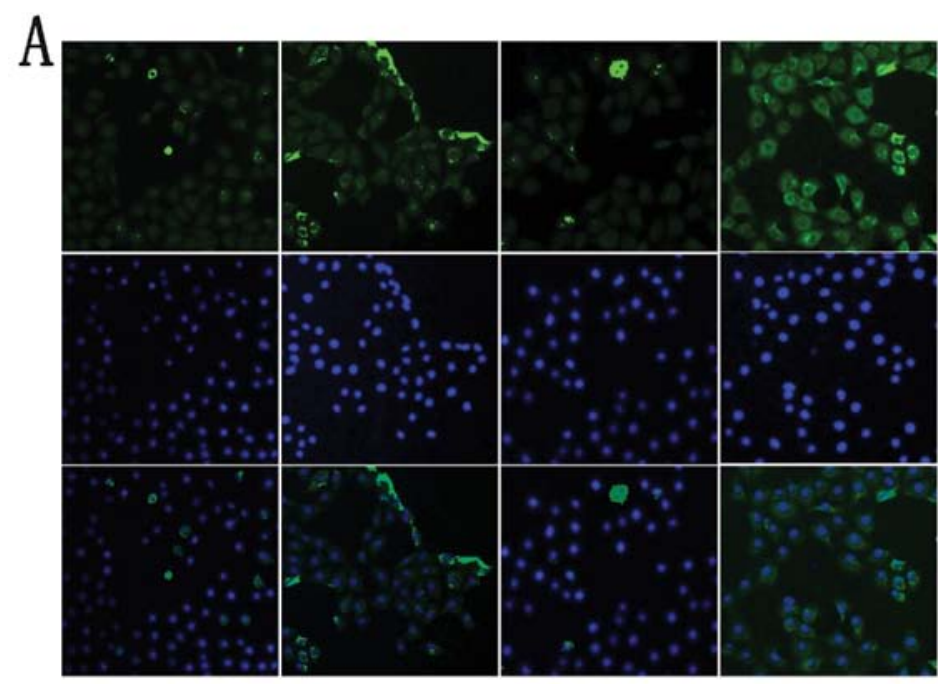

B

Control

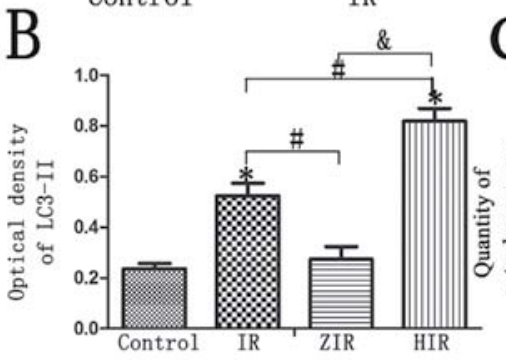

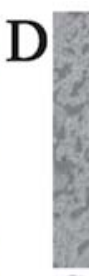
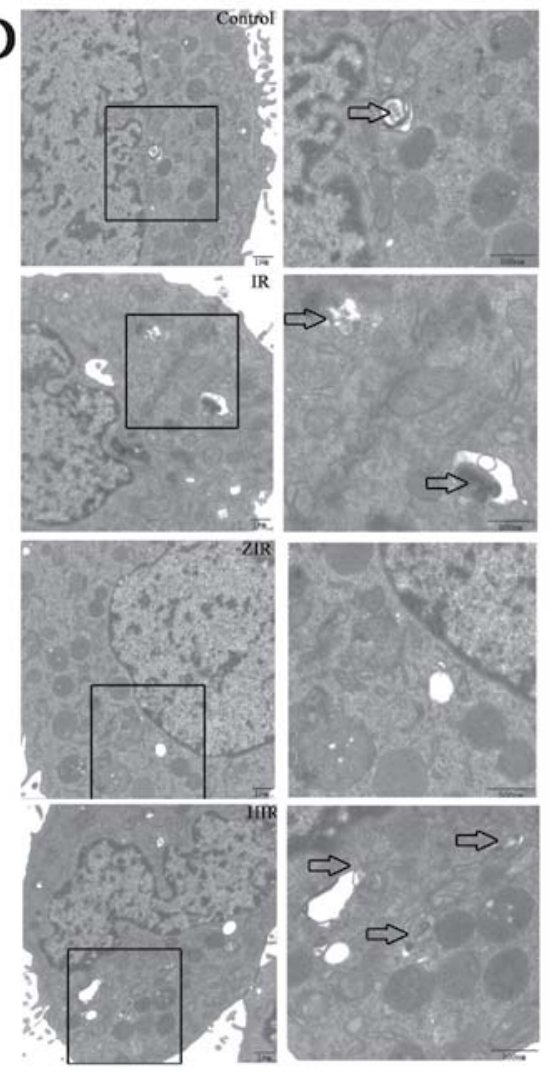

Figure 3. Treatment with zinc protoporphyrin (ZnPP) prior to ischemia/reperfusion (IR) simulation of buffalo rat liver (BRL) cells decreased light chain 3-II (LC3-II) expression, as shown by immunofluorescence and transmission electron microscopy (TEM). (A and B) Staining for LC3-II in the ZIR group was decreased, as shown by immunofluorescence, and decreased photometric values of LC3-II were observed in the ZIR group compared with the IR group; these effects were not observed in the HIR group. (C and D) Ultrastructure of BRL cells revealed by TEM showing few autophagosomes in the ZIR group compared with the IR group; the greatest number of autophagosomes was observed in the HIR group $\left({ }^{*} \mathrm{P}<0.05\right.$ compared with the control group, ${ }^{\#} \mathrm{P}<0.05$ compared with the IR group, ${ }^{\&} \mathrm{P}<0.05$ compared with the HIR group).

inhibited the expression of HO-1 in the BRL cells (Fig. 2A). Treatment with ZnPP prior to IR simulation (ZIR group) did not induce any significant differences compared with the control group (Fig. 2B; P>0.05), and this result is in agreement with the results of a previous study (31). Treatment with ZnPP prior to IR simulation in the BRL cells induced a decrease in the mRNA expression of HO-1 and LC3-II (Fig. 2C and D; $\mathrm{P}<0.05)$. HO-1 and LC3-II protein expression was inhibited in the ZIR group (Fig. 2E; $\mathrm{P}<0.05$ ).

Immunofluorescence staining revealed decreased LC3-II staining in the ZIR group (Fig. $3 \mathrm{~A}$ and $\mathrm{B} ; \mathrm{P}<0.05$ ). The ultrastructure of the BRL cells, which was examined by transmission electron microscopy (TEM), revealed few autophagosomes in the ZIR group compared with the IR group (Fig. 3C and D; $\mathrm{P}<0.05)$.

Upregulation of HO-1 induces autophagy and reduces hepatocyte apoptosis. Hemin was used in the BRL cells to induce HO-1 expression. An increase in the expression of autophagyrelated genes (LC3-II) was detected by RT-qPCR and western blot analysis (Fig. 2C-E; $\mathrm{P}<0.05$ ). Furthermore, the intensity of immunostaining for LC3-II was also significantly increased in the HIR group (Fig. 3A and B; $\mathrm{P}<0.05$ ). A greater number of autophagosomes was detected in the HIR group; this number was much greater than that detected in the control, IR and ZIR group (Fig. 3C and D; $\mathrm{P}<0.05$ ).
Annexin V/PI staining revealed that treatment with hemin decreased the early and late apoptotic rats of the BRL cells (Fig. 2B; $\mathrm{P}<0.05$ ).

The protective role of autophagy in BRL cells may be related to $\mathrm{HO}-1$ expression. In order to fiurther dertermine the role of HO-1 and autophagy, we used HBSS medium with $\mathrm{Ca}^{2+}$ and $\mathrm{Mg}^{2+}$ supplemented with $10 \mathrm{mM}$ HEPES to induce autophagy. Western blot analysis revealed the increased expression of autophagy-related genes (LC3-II) in the SIR group (Fig. 4A). In the ZSIR group, the cells were pre-treated with ZnPP prior to starvation in HBSS medium. Our results revealed that there were no significant differences in LC3-II expression between the ZIR and the ZSIR group (Fig. 4B). Additionally, there was no significant difference in the cell apoptotic rates between the ZIR and the ZSIR group (Fig. 4C).

Pre-treatment with ZnPP induces Bax, cytochrome c, caspase-3 and caspase-9 protein expression, and reduces Bcl-2 expression. As shown in Fig. 5, following treatment with mineral oil (IR group), the protein expression of Bax and cytosolic cytochrome $c$ in the BRL cells increased compared with the control group. When the cells were treated with ZnPP prior to IR simulation (ZIR group), the protein expression of Bax and cytosolic cytochrome $c$ significantly increased; however, this increase was abrogated when the cells were treated with hemin 

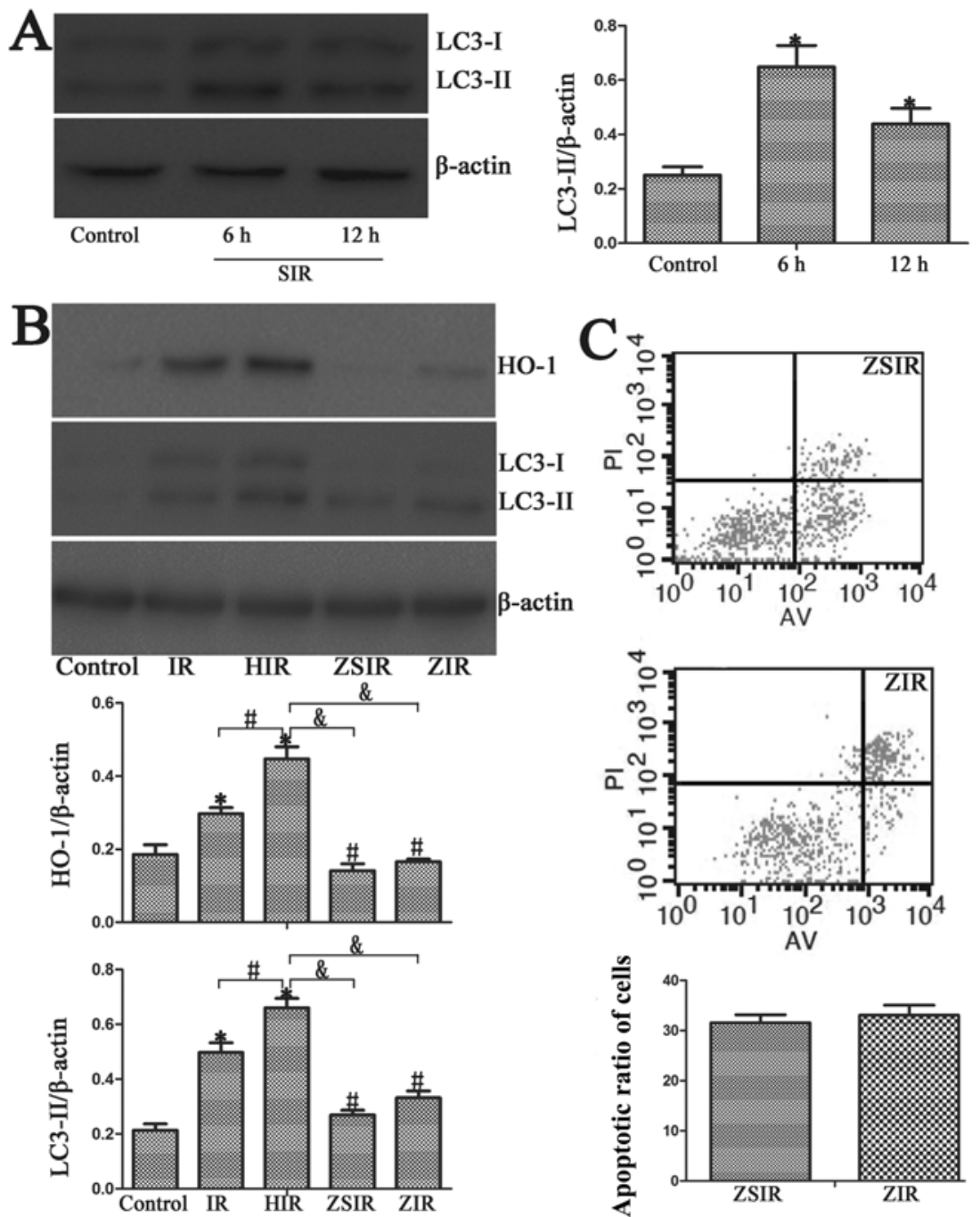

Figure 4. Heme oxygenase-1 (HO-1) and light chain 3-II (LC3-II) expression was at similar levels in the ZIR and ZSIR group. (A) Western blot analysis revealed the increased expression of autophagy-related proteins (LC3-II) in the SIR group. (B) Western blot analysis revealed that there was no significant difference in LC3-II expression between the ZIR and the ZSIR group [" $\mathrm{P}<0.05$ compared with the control group, ${ }^{\#} \mathrm{P}<0.05$ compared with the ischemia/reperfusion (IR) group, ${ }^{\&} \mathrm{P}<0.05$ compared with the HIR group]. (C) There was no significant difference in the apoptotic rates between the ZIR and ZSIR group.

prior to IR simulation (HIR group) $(\mathrm{P}<0.05)$. The expression of mitochondrial cytochrome $c$ and Bcl-2 protein was downregulated in the ZIR group compared with the IR and HIR groups $(\mathrm{P}<0.05)$.

Subsequently, the activity of cleaved caspase- 3 , caspase- 8 and caspase- 9 was detected. The expression of cleaved caspase- 3 and caspase- 9 in the cytosolic fraction at $6 \mathrm{~h}$ following IR simulation markedly increased compared with the control group (Fig. 6A and B; $\mathrm{P}<0.05$ ). In the ZIR group, the activity of cleaved caspase- 3 and capsase- 9 was increased compared to the IR group (Fig. 6A and B; $\mathrm{P}<0.05$ ). However, there was no significant change in caspase- 8 protein expression in any of the groups. Furthermore, treatment with hemin attenuated the increase in the protein expression of caspase- 3 and caspase- 9 (Fig. 6A and $\mathrm{B} ; \mathrm{P}<0.05$ ).

Higher cleaved PARP protein expression and disruption of mitochondrial membrane potential in IR and ZIR groups. Pre-treatment with ZnPP prior to IR simulation promoted the cleavage of PARP protein from its full-length form to its cleaved form. However, this was was not observed in the control and HIR group (Fig. 6A and $\mathrm{B} ; \mathrm{P}<0.05$ ).

As shown in Fig. 6C, more areas of red fluorescence were observed in the control and HIR groups. In the IR and ZIR groups however, there were more areas of green fluorescence. Greater areas of green fluorescence in cells denote a greater disruption of the mitochondrial membrane potential (Fig. 6C and $\mathrm{D} ; \mathrm{P}<0.05)$.

\section{Discussion}

Our results demonstrated that reduced autophagy was detected in an in vitro model of IR (mineral oil treatment), in which the cells were pre-treated with ZnPP. Treatment with hemin however, induced HO-1 expression in the cells and increased autophagy and alleviated hepatocyte apoptosis. Thus, autophagy induced by HBSS may be abrogated by pre-treatment with ZnPP.

Moreover, the protein expression of cytochrome $c$, Bax, Bcl-2, caspase-3, caspase- 8 and caspase- 9 was detected. The inhibition of HO-1 expression by ZnPP increased hepatocyte 


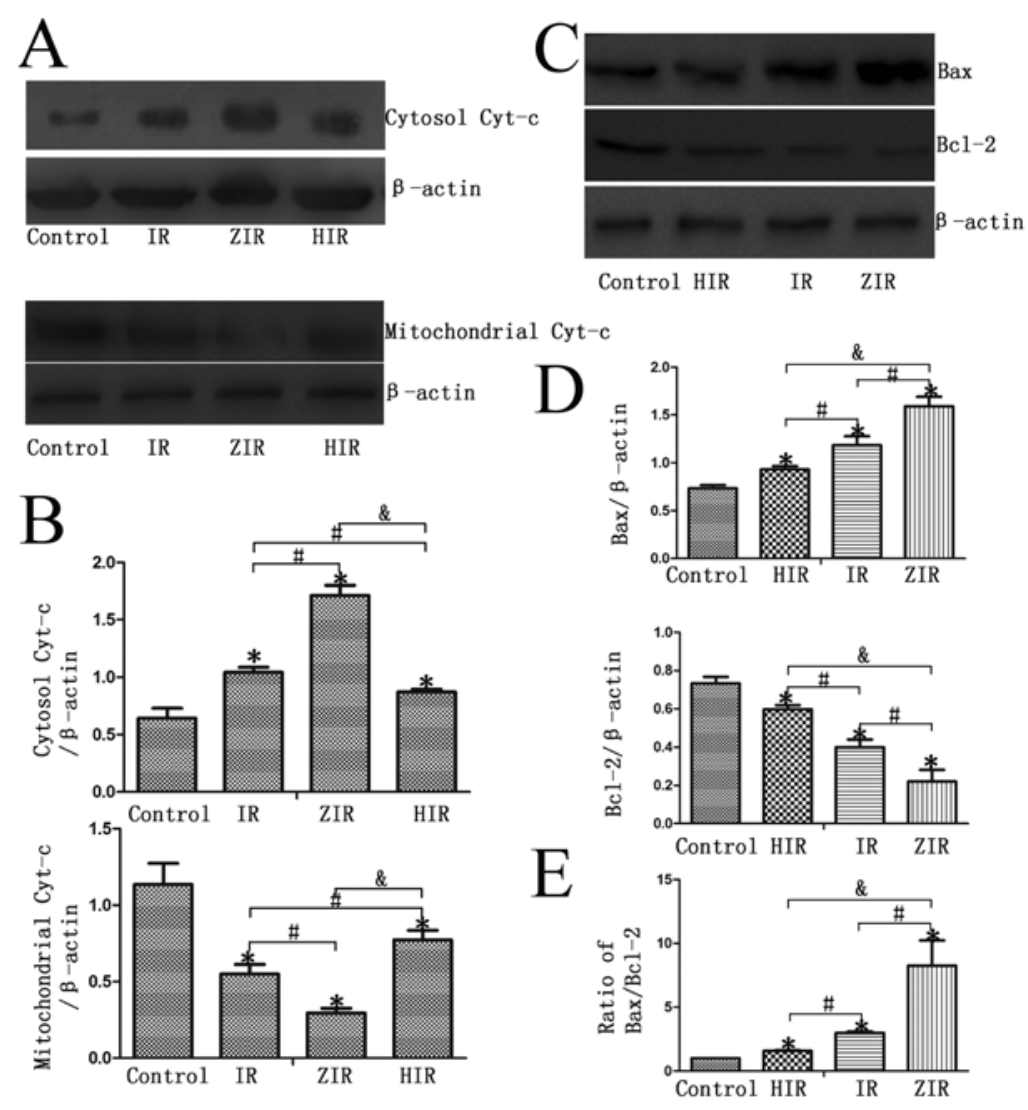

Figure 5. Pre-treatment of buffalo rat liver (BRL) cells with zinc protoporphyrin (ZnPP) induced the protein exprssion of Bax, cytosolic cytochrome $c$, while it reduced mitochondrial cytochrome $c$ and Bcl-2 expression. (A and B) Following treatment with mineral oil, the expression of cytosolic cytochrome $c$ in the BRL cells markedly increased compared with the control group. However, when the cells were pre-treated with ZnPP prior to ischemia/reperfusion (IR) simulation, the expression of cytochrome $c$ was significantly increased in the cytosolic fraction, but the increase was abrogated when hemin was used prior to IR simulation. (C and D) Bax protein expression was upregulated in the IR and ZIR groups, but Bcl-2 protein was downregulated in the IR and ZIR groups. (E) The highest ratio of $\mathrm{Bax} / \mathrm{Bcl}-2$ was observed in the ZIR group ( $\mathrm{P}<0.05$ compared with the control group, ${ }^{\#} \mathrm{P}<0.05$ compared with the IR group, ${ }^{\text {}} \mathrm{P}<0.05$ compared with the HIR group).

apoptosis, increased the protein expression of cytosolic cytochrome $c$ and Bax, and promoted the cleavage of caspase- 3 and caspase-9. The expression of mitochondrial cytochrome $c$ and Bcl-2 protein expression were decreased in the ZIR group. Based on these findings (11), as well as those of previous studies, we hypothesized that the decrease in HO-1 expression induced by ZnPP may reduce autophagy, thus increasing liver cell injury, and that this may partly occur through the activation of the mitochondrial apoptotic pathway.

Hepatic IR injury is an important phenomenon in hepatic transplantation, hepatic resection and trauma. Previous studies have identified a number of key factors associated with liver IR injury, such as Toll-like receptor (TLR), HO, leukocyte cascades and oxygen-free radicals (OFRs) $(17,18)$. Understanding the mechanism of IR injury is crucial to reducing liver injury during liver surgery. Pharmacological strategies, ischemic preconditioning or the application of new technologies have been shown to reduce liver IR injury $(19,20)$. Our results, as well as those of a previous study have shown that ischemic preconditioning induces HO-1, alleviating IR liver injury (21). As an important factor to reducing liver damage, HO-1 is a hot topic of investigation in liver IR injury.

$\mathrm{ZnPP}$ is a general metabolite formed in the process of heme biosynthesis. The lack of iron or decreased iron utilization may lead to increased ZnPP formation in the blood. Evidence suggests that increased levels of ZnPP in the blood play a role in the inhibition of $\mathrm{HO}$, which is the rate-limiting enzyme in the heme degradation pathway (9). HO is the rate-limiting enzyme in the process of the decomposition of heme metabolism, which may catabolize heme into 3 products: carbon monoxide (CO), biliverdin and free iron. There are 3 types of HO: HO-1, which mainly plays a role in the abnormal or stress state; HO-2, which is mainly distributed in the central nervous system and testes and HO-3, whose role is not known (22). Among these types, HO-1 plays a very important role in a number of organs and cells. It serves as a protective factor by as it has anti-inflammatory, anti-apoptotic and anti-proliferative properties. These beneficial effects are due to the metabolites of HO-1 (CO, biliverdin and free iron) (23-25).

Previous studies have shown that HO-1 plays a very important anti-inflammatory role in a number of inflammatory diseases, and it may act as an anti-apoptotic and anti-proliferative mediator for many damaged organs or cells under stress conditions (26-28). In liver cell injury, induced HO-1 preconditioning may lead to adaptive stress reaction, and may occur in response to organ ischemic insults, protecting the cells from injury $(29,30)$. It has been suggested that ZnPP regulates heme catabolism through the inhibition of HO-1, and thus aggravates organ damage and cell apoptosis (31). In our study, HO-1 was downregulated in the ZIR group, and higher cell apoptotic ratios were detected in this group. However, when the cells were treated with hemin prior to IR simula- 

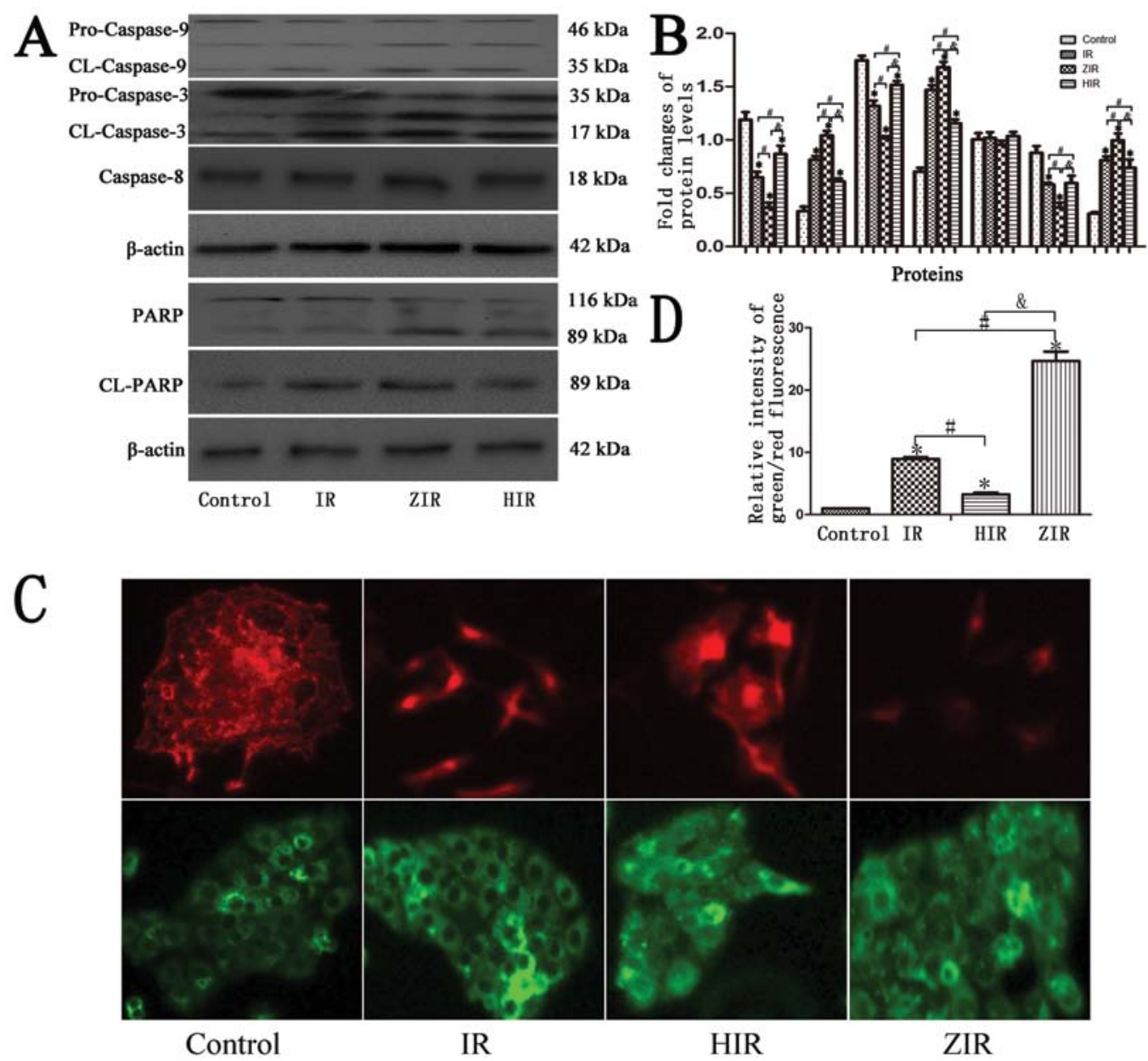

Figure 6. Pre-treatment of buffalo rat liver (BRL) cells with zinc protoporphyrin (ZnPP) induced the expression of cleaved caspase-3, caspase-9 and cleaved PARP. (A) The expression of cleaved caspase-3 and caspase-9 in the cytosolic fraction at $6 \mathrm{~h}$ after ischemia/reperfusion (IR) simulation was markedly increased compared with the control group. When compared to that in the IR group, the activity of cleaved caspase-3 and caspase- 9 was increased in the ZIR group (P<0.05). However, there were no significant changes observed in caspase- 8 expression in any of the groups. Furthermore, treatment with hemin attenuated this increase in the expression of caspase-3 and caspase-9. Treatment with ZnPP prior to IR simulation promoted the cleavage of PARP from the full-length form to its cleaved form. However, this was not observed in the control and HIR groups. (B) Fold changes of protein levels; each group of bars indicates a different protein. The order of the proteins shown is according to the order shown in (A). (C and D) More areas of red fluorescence were observed in the control and HIR group, but more areas of green fluorescence were observed in the IR and ZIR group. Higher levels of green fluorescence in cells indicate an increased disruption of mitochondrial membrane potential ( $\mathrm{P}<0.05$ compared with the control group, ${ }^{\text {"P }} \mathrm{P}<0.05$ compared with the IR group, ${ }^{\text {\& }} \mathrm{P}<0.05$ compared with the HIR group). CL, cleaved.

tion, less hepatocyte apoptosis was detected. Our results are in accordance with those of previous studies (11). The specific mechanisms involved require further investigation.

An increasing number of studies have reported that autophagy is a primarily protective factor for cells. Autophagy (self-eating) is known as a process through which cytoplasmic macromolecules or organelles are delivered to lysosomes for degradation to maintain the energy balance in the cell. Previous studies have indicated that the induction of autophagy exerts a protective effect against tissue and cell injury (32-34). Studies have demonstrated the protective effects of autophagy against liver injury $(1,6,11)$; however, the mechanisms through which autophagy prevents liver injury are not yet completely understood. Researchers have always supported the idea that one of the major functions of autophagy is to keep cells alive under stressful 'life-threatening' conditions (6,34). In our study, the lack of nutrition and oxygen to BRL cells by treatment with mineral oil increased autophagy, and this was verified by the results of RT-qPCR, western blot analsyis, electron microscopic analysis and immunofluorescence (Figs. 1-3). Thus, autophagy is an adaptive response in cells that are subjected to stress or are damaged.

Of note, in our study, the HO-1 and LC3-II proteins were simultaneously upregulated following IR simulation; when the cells were treated with $\mathrm{ZnPP}$ (inhibitor of $\mathrm{HO}-1$ ) prior to IR simulation, both proteins were simultaneously downregulated (Figs. 2 and 3). Based on the aforementioned results, we hypothesized that HO-1 may be related to autophagy. It has been demonstrated that HO-1 induces autophagy in a mouse model of liver IR injury; however, the specific mechanisms involved require further investigation (35). It has also been previously demonstrated that HO-1 protein expression is induced in injured cells or cells under stess (26-28). In our study, this was achieved by treatment with mineral oil, which caused damage to the liver cells due to the lack of nutrients and oxygen. This was also associated with the process of autophagy.

A previous study demonstrated that the phloretin-induced expression of HO-1 may contribute to the cellular defense 
mechanisms against cisplatin-induced apoptosis (36). Another study found that the induction of HO-1 ameliorated hepatocyte apoptotic activity and oxidative damage in rats with hyperthyroidism, and decreased the expression of cytochrome $c$, caspase-3, caspase- 8 and Bax. Liver injury was aggravated by the administration of $\mathrm{ZnPP}$ (HO-1 inhibitor) (37). In addition, it has been demonstrated that mitophagy, which is the selective removal of mitochondria by autophagy, plays a crucial role in IR injury (38). Thus, in the present study, we focused on HO-1, autophagy and the mitochondrial apoptotic pathway. We demonstrated the adverse effects of ZnPP on autophagy induced by treatment with mineral oil and the increase in apoptotis, which was due to the inhibition of HO-1 (Fig. 4).

There are two classical apoptosis pathways, the intrinsic pathway, which is also known as the mitochondrial-mediated pathway and the extrinsic or death receptor-mediated pathway (39). It has also been reported that the Bcl-2 family of proteins, including the anti-apoptotic protein, Bcl-2, and the pro-apoptotic protein, Bax, are crucial in initiating the mitochondrial death cascade. When cells are under stress, Bax protein translocates to the outer mitochondrial membrane and promotes the release of cytochrome $c$ from the mitochondria to the cytosol. By contrast, $\mathrm{Bcl}-2$ protein disrupts this process and decreases apoptosis (40). It has also been demonstrated that $\mathrm{Bcl}-2$ expression is reduced while that of Bax is increased in HECI-OC1 cells pre-treated with ZnPP (36). Thus, we hypothesized that pre-treatment with ZnPP would promote the release of cytochrome $c$ and the activation of the mitochondrial death cascade. The upregulation of Bax and the downregulation of Bcl-2 was observed in the ZIR group, while the induction of HO-1 prevented this event. The expression of cytosolic cytochrome $c$ was induced in the cells pre-treated with ZnPP and the expression of mitochondrial cytochrome $c$ was decreased. When mitochondrial membrane potential was examined, more areas of red fluorescence were observed in the control and the HIR group; however, more areas of green fluorescence were observed in the IR and ZIR group. Higher levels of green fluorescence indicate a greater disruption of mitochondrial membrane potential. A previous study found that the activation of caspase- 3 and caspase- 9 was important in the intrinsic pathway (41). In our study, there was an increase in the expression of cleaved caspase- 3 and caspase- 9 in the IR group and the group treated with ZnPP prior to IR simulation; however, this increase was not observed in the control and HIR group.

PARP protein, another important protein associated with apoptosis, is cleaved by activated caspase-3. It is regarded as the symbol of the activation of caspase-3 (42). In our study, the cleavage of PARP from the full-length $116 \mathrm{kDa}$ form to its cleaved $89 \mathrm{kDa}$ form was observed in the IR and ZIR groups (Fig. 6). Based on this result, we hypothesized that the inhibition of HO-1 by ZnPP increased BRL cell apoptosis and that it may play a role in the mitochondrial-mediated apoptotic pathway.

In conclusion, in the present study, we demonstrate that $\mathrm{ZnPP}$ reduces $\mathrm{HO}-1$ expression and subsequently inhibits autophagy, thus aggravating BRL cell IR injury and has a pro-apoptotic effect via the mitochondrial apoptotic pathway. In the present study, we hypothesized that the reduction of autophagy by HO-1 inhibition may lead to the activation of the mitochondrial apoptotic pathway. However, the association between autophagy and the mitochondrial apoptotic pathway, as well as the role of autophagy in $\mathrm{ZnPP}$-induced apoptosis require further investigation.

\section{Acknowledgements}

The study was supported by grants from the National Natural Science Foundation of China (81170415), Nanjing Medical University (2012NJMU087) and Xuzhou City Central Hospital (XZS2013018).

\section{References}

1. Rautou PE,Mansouri A,LebrecD,DurandF, Valla D and Moreau R: Autophagy in liver diseases. J Hepatol 53: 1123-1134, 2010.

2. Nivon M, Richet E, Codogno P, Arrigo AP and Kretz-Remy C: Autophagy activation by NFkappaB is essential for cell survival after heat shock. Autophagy 5: 766-783, 2009.

3. Mizushima N, Levine B, Cuervo AM and Klionsky DJ: Autophagy fights disease through cellular self-digestion. Nature 451: 1069-1075, 2008.

4. Teoh NC: Hepatic ischemia reperfusion injury: contemporary perspectives on pathogenic mechanisms and basis for hepatoprotection-the good, bad and deadly. J Gastroenterol Hepatol 26 (Suppl 1): S180-S187, 2011.

5. Walsh KB, Toledo AH, Rivera-Chavez FA, Lopez-Neblina F and Toledo-Pereyra LH: Inflammatory mediators of liver ischemia-reperfusion injury. Exp Clin Transplant 7: 78-93, 2009.

6. Esposti DD, Domart MC, Sebagh M, Harper F, Pierron G, Brenner C and Lemoine A: Autophagy is induced by ischemic preconditioning in human livers formerly treated by chemotherapy to limit necrosis. Autophagy 6: 172-174, 2010.

7. Qu X,Zou Z, Sun Q, Luby-Phelps K, Cheng P, Hogan RN, Gilpin C and Levine B: Autophagy gene-dependent clearance of apoptotic cells during embryonic development. Cell 128: 931-946, 2007.

8. Hara T, Nakamura K, Matsui M, et al: Suppression of basal autophagy in neural cells causes neurodegenerative disease in mice. Nature 441: 885-889, 2006.

9. Labbé RF, Vreman HJ and Stevenson DK: Zinc protoporphyrin: a metabolite with a mission. Clin Chem 45: 2060-2072, 1999.

10. Kim SJ, Eum HA, Billiar TR and Lee SM: Role of heme oxygenase 1 in TNF/TNF receptor-mediated apoptosis after hepatic ischemia/reperfusion in rats. Shock 39: 380-388, 2013.

11. Carchman EH, Rao J, Loughran PA, Rosengart MR and Zuckerbraun BS: Heme oxygenase-1-mediated autophagy protects against hepatocyte cell death and hepatic injury from infection/sepsis in mice. Hepatology 53: 2053-2062, 2011.

12. Meldrum KK, Burnett AL, Meng X, Misseri R, Shaw MB, Gearhart JP and Meldrum DR: Liposomal delivery of heat shock protein 72 into renal tubular cells blocks nuclear factor-kappaB activation,tumor necrosis factor-alpha production, and subsequent ischemia-induced apoptosis. Circ Res 92: 293-299, 2003.

13. Meldrum KK, Meldrum DR, Hile KL, et al: p38 MAPK mediates renal tubular cell TNF-alpha production and TNF-alpha-dependent apoptosis during simulated ischemia. Am J Physiol Cell Physiol 281: C563-C570, 2001.

14. Choi BM,Pae HO, Kim YM and Chung HT: Nitric oxide-mediated cytoprotection of hepatocytes from glucose deprivation-induced cytotoxicity involvement of heme oxygenase-1. Hepatology 37 : 810-823, 2003.

15. Settembre C, Di Malta C, Polito VA, et al: TFEB links autophagy to lysosomal biogenesis. Science 332: 1429-1433, 2011.

16. Lewis JS, Meeke K, Osipo C, et al: Intrinsic mechanism of estradiol-induced apoptosis in breast cancer cells resistant to estrogen deprivation. J Natl Cancer Inst 97: 1746-1759, 2005.

17. Serizawa A, Nakamura S, Suzuki S, Baba S and Nakano M: Involvement of platelet-activating factor in cytokine production and neutrophil activation after hepatic ischemia-reperfusion. Hepatology 23: 1656-1663, 1996.

18. Vardanian AJ, Busuttil RW and Kupiec-Weglinski JW: Molecular mediators of liver ischemia and reperfusion injury: a brief review. Mol Med 14: 337-345, 2008.

19. Selzner N, Rudiger H, Graf R and Clavien PA: Protective strategies against ischemic injury of the liver. Gastroenterology 125: 917-936, 2003. 
20. Shao CH, Chen SL, Dong TF, et al: Transplantation of bone marrow-derived mesenchymal stem cells after regional hepatic irradiation ameliorates thioacetamide-induced liver fibrosis in rats. J Surg Res 186: 408-416, 2014.

21. Wang CF, Wang ZY, Tao SF, Ding J, Sun LJ, Li JY and Quan ZW: Preconditioning donor liver with Nodosin perfusion lessens rat ischemia reperfusion injury via heme oxygenase-1 upregulation. J Gastroenterol Hepatol 27: 832-840, 2012.

22. Tsuchihashi S, Fondevila C and Kupiec-Weglinski JW: Heme oxygenase system in ischemia and reperfusion injury. Ann Transplant 9: 84-87, 2004.

23. Otterbein LE, Soares MP, Yamashita K and Bach FH: Heme oxygenase-1: unleashing the protective properties of heme. Trends Immunol 24: 449-455, 2003.

24. Liu FC, Yu HP, Hwang TL and Tsai YF: Protective effect of tropisetron on rodent hepatic injury after trauma-hemorrhagic shock through P38 MAPK-dependent hemeoxygenase-1 expression. PLoS One 7: e53203, 2012.

25. Liu FC, Hwang TL, Lau YT and Yu HP: Mechanism of salutary effects of astringinin on rodent hepatic injury following trauma-hemorrhage: Akt-dependent hemeoxygenase-1 signaling pathways. PLoS One 6: e25907, 2011.

26. Mandal P, Pritchard MT and Nagy LE: Anti-inflammatory pathways and alcoholic liver disease: role of an adiponectin/interleukin-10/heme oxygenase-1pathway. World J Gastroenterol 16: 1330-1336, 2010.

27. Öllinger R and Pratschke J: Role of heme oxygenase-1 in transplantation. Transpl Int 23: 1071-1081, 2010.

28. Mandal P, Park PH, McMullen MR, Pratt BT and Nagy LE: The anti-inflammatory effects of adiponectin are mediated via a heme oxygenase-1-dependent pathway in rat Kupffer cells. Hepatology 51: 1420-1429, 2010.

29. Devey L, Ferenbach D, Mohr E, Sangster K, Bellamy CO, Hughes $J$ and Wigmore SJ: Tissue-resident macrophages protect the liver from ischemia reperfusion injury via a heme oxygenase-1-dependent mechanism. Mol Ther 17: 65-72, 2009.

30. Mashreghi MF, Klemz R, Knosalla IS, et al: Inhibition of dendritic cell maturation and function is independent of heme oxygenase 1 but requires the activation of STAT3. J Immunol. 180: 7919-7930, 2008.

31. Wu SY, Li MH, Ko FC, Wu GC, Huang KL and Chu SJ: Protective effect of hypercapnic acidosis in ischemia-reperfusion lung injury is attributable to upregulation of heme oxygenase-1. PLoS One 8: e74742, 2013.
32. Yan W, Zhang H, Bai X, Lu Y, Dong H and Xiong L: Autophagy activation is involved in neuroprotection induced by hyperbaric oxygen preconditioning against focal cerebral ischemia in rats. Brain Res 1402: 109-121, 2011.

33. Reed M, Morris SH, Jang S, Mukherjee S, Yue Z and Lukacs NW: Autophagy-inducing protein beclin-1 in dendritic cells regulates CD4 $\mathrm{T}$ cell responses and disease severity during respiratory syncytial virus infection. J Immunol 191: 2526-2537, 2013.

34. Chen YY, Sun LQ, Wang BA, Zou XM, Mu YM and Lu JM: Palmitate induces autophagy in pancreatic $\beta$-cells via endoplasmic reticulum stress and its downstream JNK pathway. Int J Mol Med 32: 1401-1406, 2013.

35. Wang Y, Shen J, Xiong X, et al: Remote ischemic preconditioning protects against liver ischemia-reperfusion injury via heme oxygenase-1-induced autophagy. PLoS One 9: e98834, 2014.

36. Choi BM, Chen XY, Gao SS, Zhu R and Kim BR: Anti-apoptotic effect of phloretin on cisplatin-induced apoptosis in HEI-OC1 auditory cells. Pharmacol Rep 63: 708-716, 2011

37. Giriş M, Erbil Y, Depboylu B, Mete O, Türkoğlu U, Abbasoğlu SD and Uysal M: Heme oxygenase-1 prevents hyperthyroidism induced hepatic damage via an antioxidant and antiapoptotic pathway. J Surg Res 164: 266-275, 2010.

38. Kim I and Lemasters JJ: Mitophagy selectively degrades individual damaged mitochondria after photoirradiation. Antioxid Redox Signal 14: 1919-1928, 2011.

39. Xie SQ, Zhang YH, Li Q, Xu FH, Miao JW, Zhao J and Wang CJ: 3-Nitro-naphthalimide and nitrogen mustard conjugate NNM-25 induces hepatocellular carcinoma apoptosis via PARP-1/p53 pathway. Apoptosis 17: 725-734, 2012.

40. Qi F, Inagaki Y, Gao B, et al: Bufalin and cinobufagin induce apoptosis of human hepatocellular carcinoma cells via Fas- and mitochondria-mediated pathways. Cancer Sci 102: 951-958, 2011.

41. Nicholson DW: From bench to clinic with apoptosis-based therapeutic agents. Nature 407: 810-816, 2000.

42. Scovassi AI and Poirier GG: Poly (ADP-ribosylation) and apoptosis. Mol Cell Biochem 199: 125-137, 1999. 